Document downloaded from:

http://hdl.handle.net/10251/63689

This paper must be cited as:

Marquez-Barja, JM.; Ahmadi, H.; Tornell, SM.; Tavares De Araujo Cesariny Calafate, CM.; Cano Escribá, JC.; Manzoni, P.; Dasilva, LA. (2015). Breaking the Vehicular Wireless Communications Barriers: Vertical Handover Techniques for Heterogeneous Networks. IEEE Transactions on Vehicular Technology. 64(12):5878-5890. doi:10.1109/TVT.2014.2386911.

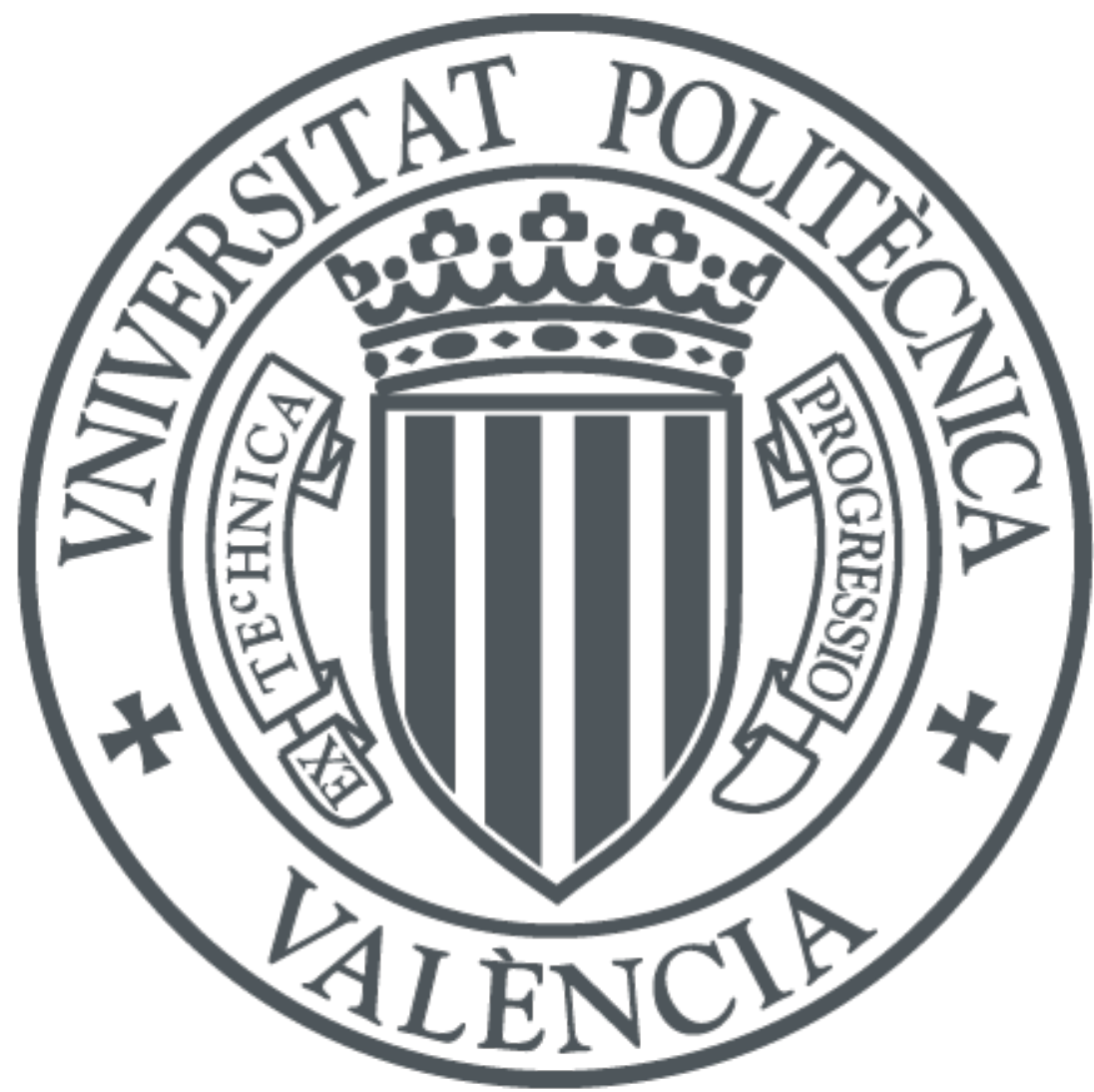

The final publication is available at

http://dx.doi.org/ 10.1109/TVT.2014.2386911

Copyright Institute of Electrical and Electronics Engineers (IEEE)

Additional Information

"(C) 2014 IEEE. Personal use of this material is permitted. Permission from IEEE must be obtained for all other uses, in any current or future media, including reprinting/republishing this material for advertising or promotional purposes, creating new collective works, for resale or redistribution to servers or lists, or reuse of any copyrighted component of this work in other works." 


\title{
Breaking the vehicular wireless communications barriers: Vertical handover techniques within heterogeneous networks
}

\author{
Johann M. Marquez-Barja, Member, IEEE, Hamed Ahmadi, Member, IEEE, Sergio M. Tornell, Carlos T. Calafate, \\ Juan-Carlos Cano, Pietro Manzoni, Member, IEEE, and Luiz A. DaSilva, Senior Member, IEEE
}

\begin{abstract}
End-users increasingly expect ubiquitous connectivity while on the move. With a variety of wireless access technologies available, we expect to always be connected to the technology that best matches our performance goals and price points. Meanwhile, sophisticated on-board units enable geolocation and complex computation in support of handover. In this paper, we present an overview of vertical handover techniques, and propose an algorithm empowered by the IEEE 802.21 standard, that considers the particularities of the vehicular networks, the surrounding context, the application requirements, the user preferences, and the different available wireless networks (i.e., Wi-Fi, WiMAX and UMTS) in order to improve users' quality of experience. Our results demonstrate that our approach, under the considered scenario, is able to meet application requirements while ensuring user preferences are also met.
\end{abstract}

Index Terms - Vehicular network, vertical handover, MCDM, heterogeneous networks, IEEE 802.21, Wi-Fi, WiMAX, UMTS, ns-2

\section{INTRODUCTION}

Vehicles are constantly being improved by enhancing mechanical performance, as well as comfort and safety. Wireless communications, including on-board "anywhere, anytime" communication, boosts the vehicle's features by reinforcing safety, and by offering new services such as infotainment and in-car connectivity.

Nowadays, the automotive industry is manufacturing vehicles with On-Board Units (OBUs) containing several communication interfaces such as Wireless Fidelity (Wi-Fi), Universal Mobile Telecommunications System (UMTS), Bluetooth, Near Field Communication (NFC) and even some prototypes with Worldwide interoperability for Microwave Access (WiMAX), as well as improved Global Positioning System (GPS) receivers. With the combined use of such resources, the communication demand of the end-users within vehicular networks is evolving from short safety messages towards online multimedia sessions. To meet these new end-users' demands and improve their Quality of Experience (QoE), connectivity should be guaranteed with an adequate Quality of Service (QoS).

Johann Marquez-Barja and Hamed Ahmadi are with CTVR, the telecommunications research centre, Trinity College Dublin, Ireland, e-mail: marquejm@tcd.ie

Sergio M. Tornell, Carlos T. Calafate, Juan-Carlos Cano and Pietro Manzoni are with the Universitat Politècnica de València, Spain.

Luiz A. DaSilva is with CTVR, the telecommunications research centre, Trinity College Dublin, Ireland, and also with Virginia Tech, USA.

Manuscript received xxxx; revised December xxxx.

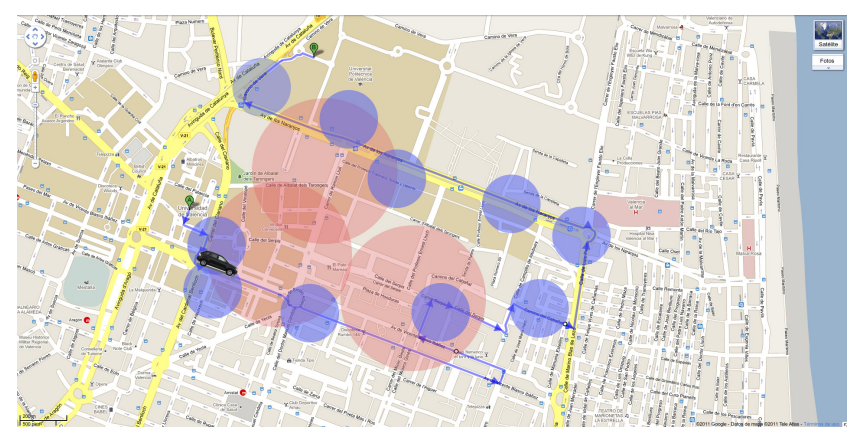

Fig. 1. Example of a pathway with different wireless network coverage areas.

Current outdoor wireless communication technologies offer solutions which differ in aspects such as coverage, data rate, frequency and modulation. The heterogeneity of such technologies, rather than being a pitfall for vehicular communications, should be seen as an advantage, since vehicles can make the most out of the diverse wireless technologies in order to mantain continuous communication while journeying from one location to another. Figure 1 illustrates a particular vehicular itinerary within an urban environment with heterogeneous wireless access coverage, with correspondingly different ranges.

Within Vehicular Networks (VNs) a vehicle is considered as a node of the network, being equipped with multiple interfaces that provide access to different technologies such as GPS, Wi-Fi, WiMAX, UMTS and Long Term Evolution (LTE). Vehicles are able to communicate among themselves and with their Point of Attachment (PoA) [Access Points (APs) or Basestations (BSs)] under the ad-hoc or the infrastructure modes [1], respectively. The vehicular contexts are, from a wireless communications point of view, highly dynamic, and vehicles must be able to deal with heterogeneity through context awareness and Vertical Handover (VHO) capabilities. To provide context awareness, the vehicles and the networking elements (e.g., BSs or APs) should offer useful information about the status of the network, geolocation, the network provider assets, and their specifications. Moreover, vehicles should offer information not only about their technological capabilities, but also relevant information in terms of user preferences. Regarding VHO, the elements of the network should integrate the IEEE 802.21 standard primitives [2] to enable the capabilities of the Media Independent Handover 
Function (MIHF) protocol in order to provide a homogeneous interface for seamless handovers among heterogeneous wireless networks (e.g., Wi-Fi, WiMAX, UMTS, LTE). Furthermore, in order to choose the most suitable Candidate Network (CN) - from the set of available heterogeneous wireless access networks - that fulfils the QoS connectivity requirements, a decision-making process must be performed considering several context factors, as well as the performance of the different networks; this process must be accurate and fast to avoid negatively impacting connectivity or QoE.

In this paper, we present a Vertical Handover Decision Algorithm (VHDA) designed for VNs that falls under the infrastructure mode category, i.e., AP-based communications rather than the traditional ad-hoc mode, the so-called Vehicular Ad-hoc Networks (VANETs). The VHDA proposed is empowered by the IEEE 802.21 standard. Our proposed solution, in order to select a $\mathrm{CN}$ to hand over to, considers several factors such as the geolocation coordinates, driving itinerary (the route to reach location B from location A), map layouts (the road directions and layout), user preferences regarding trade-offs between price and network performance, and surrounding heterogeneous wireless networks (available wireless and mobile networks). Moreover, the decision-making process proposed is based on a Multiple Criteria DecisionMaking (MCDM) algorithm that selects the network that best meets the end-user connectivity requirements, the VHDA is also extensible to other highly dynamic mobile networks.

\section{RELATED WORK}

\section{A. Vertical handover in heterogeneous networks}

Stemm and Katz [3] proposed one of the first vertical handover schemes by allowing handovers among the IBM Infrared Wireless LAN, the AT\&T WaveLAN and the Metricom Ricochet Network, in-building, campus, and wide area wireless technologies, respectively, that were available in those days. Their proposal also took into account Mobile IP (MIP) and routing for mobility issues. This early work set the stage for dealing with heterogeneous networks through vertical handover methods.

In the past few years, with the advent of new wireless technologies, several works have tackled VHO among a wide variety of wireless technologies such as UMTS, Wi-Fi, LTE, Wireless Broadband (WiBro), ZigBee, Radio Frequency Identification (RFID), Bluetooth (BT), Digital Video Broadcasting (DVB), and Multimedia Broadcast/Multicast Service (MBMS), or even Low Earth Orbit (LEO) satellite [4]. Most proposals consider a wireless environment where the User Equipment (UE) is a mobile phone or laptop within a pedestrian mobility model, or scenarios with low mobility. Moreover, most proposals evaluate the VHO using only two technologies (usually Wi-Fi and UMTS), and only some works have considered three or more technologies [4].

Over the same period, vehicular communications have been improved by adding short- and long-distance communication devices, GPS, and sensing systems to vehicles. All these communication capabilities work under highly dynamic vehicular scenarios.
The use of GPS information to improve handover and the network selection process, in the scope of a single type of wireless network, has been widely studied [5], [6], [7]. Geolocation information can also be applied to improve the decisionmaking process to hand over among heterogeneous networks. Ylianttila et al. [8] presented one of the first approaches, using GPS to manage the current location of the mobile device. Their proposal considered the handover scenario under Wi-Fi and UMTS cells. The authors performed the decisionmaking process by considering the Received Signal Strength (RSS) of the CNs. Using GPS information (coordinates, speed, direction), the mobility prediction can be improved, and a couple of works take advantage of it to improve the VHO process by predicting the path and the next most likely PoA within the path [9], [10]. Wang et al. [11] present a VHO method that considers several factors such as RSS, data rate, Bit Error Rate (BER), and movement trend; in order to select the network that best suits the prioritized decision parameter, this method relies on a decision-tree where, depending on the parameter selected at each decision event (node), the decision-process may continue or not through that branch. Moreover, this solution considers 3G, WiMAX and the IEEE $802.11 \mathrm{p}$ as underlying connectivity technologies. However, the solution does not consider the IEEE 802.21 as part of the VHO framework, having to deploy a customized solution to provide communication among the different network interfaces and network entities. Wang et al. [12] considers also WiMAX as part of the underlying connectivity, along with Wi-Fi. The authors take into account the particularities of the governing protocols, such as awakening times, sleep modes, and Protocol Data Units (PDUs) to enable the decision-making process. When this contribution was made, IEEE 802.21 had not been released; therefore, the authors did count with such a flexible tool when dealing with heterogeneous networks. So far there have been works that focus on the decisionmaking process by relying on fuzzy logic [13], [14], or Multiple Attribute Decision-Making (MADM) [15], [16], [17] techniques, taking into account several factors such as RSS, mobility, speed, distance among the APs, geolocation, and data rates. Nevertheless, these works focus solely on the decisionmaking process, and do not take into account the IEEE 802.21 standard to perform not only the decision-making process, but also the supporting processes such as gathering/updating information, the VHO itself, and managing the data flows among network interfaces.

\section{B. A protocol for handovers in heterogeneous networks}

Since 2004, the IEEE 802.21 Working Group has been working on the Media Independent Handover Services Protocol [2], whose purpose is to provide a homogeneous functioninterface between heterogeneous network technologies. Currently, there are works addressing the performance of the IEEE 802.21 technology [18], as well as real implementations on operating systems, smartphones [19], and tablet devices [20].

The IEEE 802.21 standard specifies media accessindependent mechanisms that optimize handovers among heterogeneous IEEE 802 systems as well as cellular systems. The 

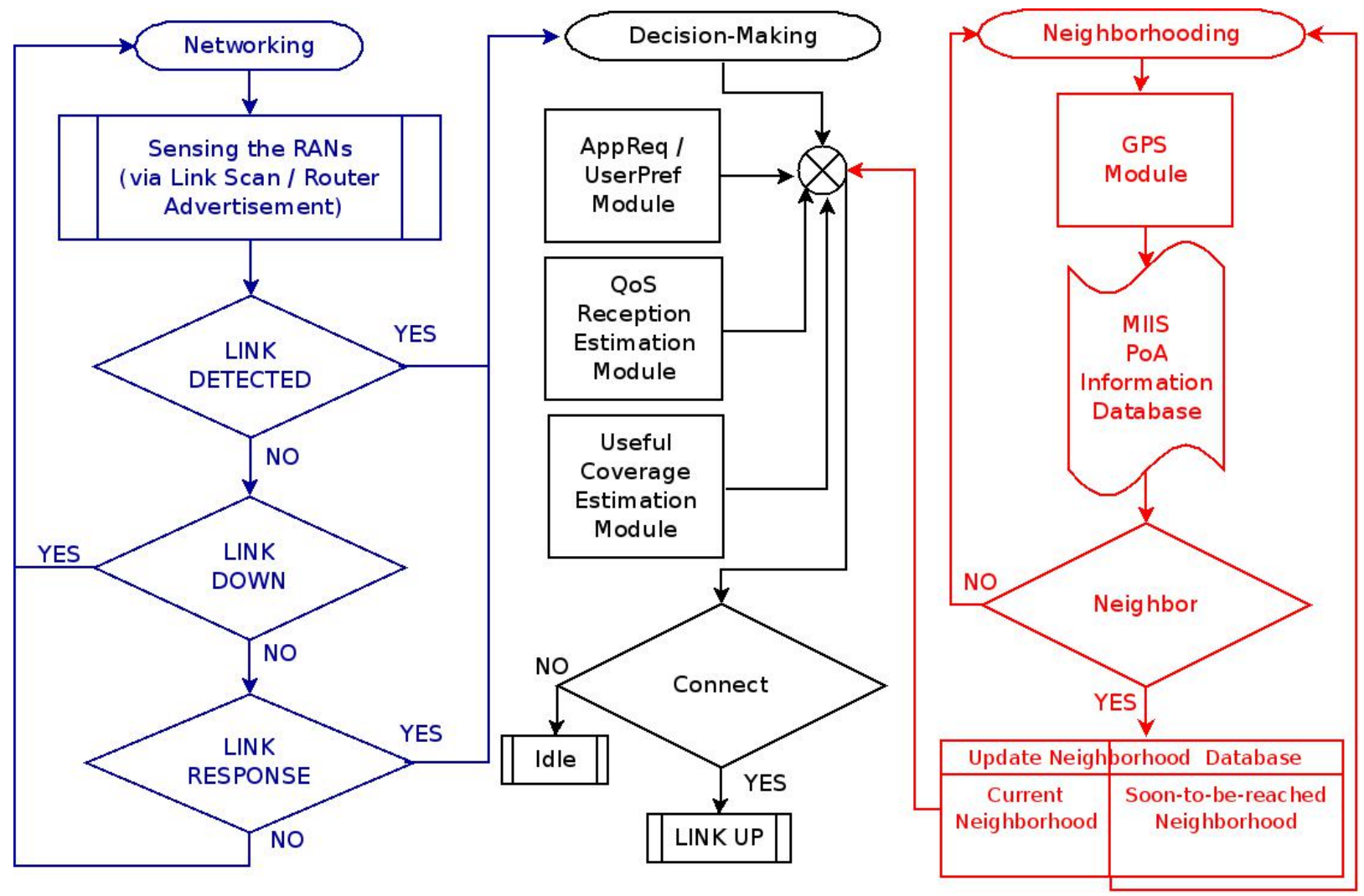

Fig. 2. Neighborhood Aware vehIculaR Handover Algorithm (NAIRHA).

standard defines the Media Independent Handover Function (MIHF) protocol, which describes the messages exchanged between peer Media Independent Handover (MIH) entities, offering a common message payload across different technologies $(802.3,802.11,802.16$, and cellular). The basic services offered by the MIHF are the Media Independent Event Service (MIES), the Media Independent Information Service (MIIS), and the Media Independent Command Service (MICS). Each service interacts, via messages, with the upper and lower layers.

The MIES detects the changes in the lower layers, e.g., changes in the physical channel conditions. The MIHF notifies events occurring in the lower layers to the Media Independent Handover Users (MIHUs) as they have requested. The MIES covers events such as: state change events (link up, link down, link parameter changes); predictive events (link going down); and network-initiated events (load balancing, operator preferences). The MIIS allows the MIHF to discover its network environment by gathering information that the upper layers use to make decisions. The information elements refer to the list of available networks, location of PoA, operator ID, roaming partners, cost, security, QoS, PoA capabilities, and vendor-specific information.

Finally, the MICS allows the MIHU to take control over the lower layers through a set of commands. With the information gathered by the MIES and MIIS, the MIHU decides whether to switch from one PoA to another. The commands allow the handover entity not only to execute the handover, but also to set different parameters in the lower layer elements.
To our knowledge, within the vehicular networks field there are no VHO works taking into account the context and the route information, based on: geolocation; the use of Wi-Fi, WiMAX, and UMTS as underlying wireless technologies; the network status; the user profiles (preferences); the running application requirements; and the homogeneous management for heterogeneous networks, based on the IEEE 802.21 standard. Our paper studies the VHO process considering all the above factors.

\section{SMART NEIGHBOURHOOD-AWARE DECISION ALGORITHM}

In this section we will describe the Neighborhood Aware vehIculaR Handover Algorithm (NAIRHA), an enhanced VHDA designed for VNs, that takes into consideration the surrounding context, different available types of wireless networks, networking elements information, geolocation features (location and navigation), user preferences and application requirements, in order to select the most suitable CN. NAIRHA makes use of the IEEE 802.21 standard, using the MIIS to collect networking information, the MICS to interact with the different network interfaces, and the MIES to sense the state of the networks. Moreover, the location and navigation information enhances the surrounding context data by allowing mobile devices to continuously gather information from the current and soon-to-be-reached neighborhoods. Concerning the decision-making process, NAIRHA uses the Simple Additive Weighting (SAW) algorithm - an MCDM algorithm - 
to fairly evaluate the candidates and choose the most suitable one that meets the multiple requirements defined.

NAIRHA has several modules residing on the OBU, grouped into three sets of tasks (Neighborhooding, DecisionMaking and Networking) that perform different duties in order to achieve seamless handover to the most suitable CN. Also, NAIRHA is able to take advantage of the features of the current OBUs offered by the automotive industry ${ }^{1}$ as well as the OBUs based on smartphones or arduino-type devices [21], such as multiple networking interfaces, GPS information, maps and routes, without having strict energy constrains due to the continuous power supply in the vehicles. Figure 2 shows the flow diagram of NAIRHA. We now proceed to describe the main components of the algorithm.

\section{A. Neighborhooding task components}

One of the features of NAIRHA is the introduction of the neighborhood concept, which is the use of the surrounding context information based on the geolocation of the vehicle while it is moving within a navigation route. Basically, a neighborhood is a collection of information related to the surrounding heterogeneous networks and their connectivity elements for a given location. To manage a neighborhood, the following modules are required:

1) GPS module: This module is in charge of two main duties: i) navigation route calculation; and ii) geolocation calculation. This module can be queried at any time by other modules, providing as an output the current geolocation, the route to reach a certain location, and/or the future geolocation if the itinerary is followed at the current average speed (considering the recent speed history).

2) Neighborhood database: The database stores information in the OBU regarding the current and soon-to-be-reached neighborhoods. The MIIS service is used to retrieve information from the different MIIS databases located at different points of the network. The information retrieved includes the ID of the network, the ID of the PoA, its geolocation, coverage, monetary cost per MB of data delivered, nominal data rate offered, and the data rate achieved by the most recent set of users [2]. Concerning the soon-to-be-reached neighborhood, the information retrieved is related to the PoAs that will be part of the surrounding context in the near future. Depending on the frequency with which the neighborhood is being updated, and to how much distance is being considered in advance from the location sample, the current and the soonto-be-reached neighborhood could store the same or different information. Therefore, retrieving the information and updating the neighborhood database with consistent information is a crucial process.

The Sensing Period (SP) defines how frequently the neighborhood database must be updated by querying the GPS module and by querying the MIIS databases; such queries are performed by the OBU. The Prediction Window (PW) is a period of time that is translated into the distance within the

\footnotetext{
${ }^{1}$ http://www.mobility.siemens.com/mobility/global/SiteCollectionDocuments/ en/road-solutions/interurban/tolling-systems-for-freeways/Sitraffic-SensusUnit-en.pdf
}

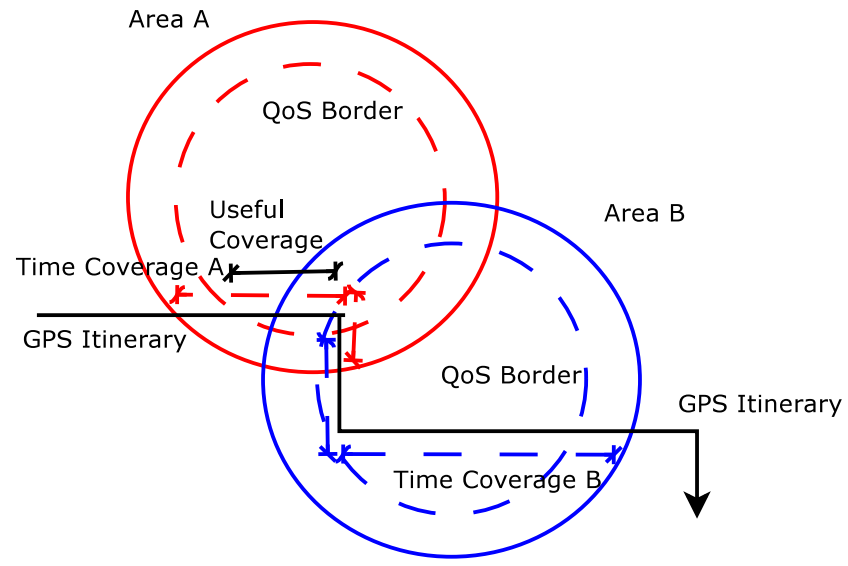

Fig. 3. Navigation itinerary, cell and useful coverage, and QoS border.

path that will be covered during such time. Summarizing, the SP is related to how fresh the data stored in the neighborhood is, while the PW is related on how accurate this information is, since a bigger PW will tend to be associated with a higher probability of error than a small PW. We shall achieve a good trade-off between SP and PW, and the calculation of desirable SP and PW is presented in Section III-C2.

When establishing a neighborhood, to determine whether a PoA is within the neighborhood, the Haversine formula [22] is used to compute the geodistance from the current geolocation of the vehicle to the geolocation of each PoA discovered by querying the MIIS databases. The geodistance is calculated as:

$$
\mathrm{d}=2 \mathrm{R} \arcsin \left(\sqrt{\sin ^{2}\left(\frac{\triangle \varphi}{2}\right)+\cos \left(\varphi_{\text {Vehicle }}\right) \cos \left(\varphi_{\mathrm{POA}}\right) \sin ^{2}\left(\frac{\triangle \lambda}{2}\right)}\right),
$$

where $\mathrm{R}$ is the mean radius of the Earth, $\varphi$ denotes latitude and $\lambda$ denotes longitude. The difference of the latitude between the geolocation of the vehicle and the PoA is denoted by $\triangle \varphi$, and the difference regarding the longitude by $\triangle \lambda$.

Each entry in the neighborhood database stores the features of each PoA that is within the neigborhood, as well as the Useful Coverage Time (UCT) for the PoA. The latter time is calculated considering several factors under the cell coverage, as explained in the following section.

3) Useful coverage time (UCT): The UCT is the time that the mobile spends within the coverage area of a cell while able to obtain the peak data rate from that cell. This time may vary due to several issues such as whether the itinerary tangentially crosses the coverage area, or the existence of overlapping coverage areas along the itinerary path, as illustrated in Figure 3. Moreover, the UCT may also vary due to QoS fluctuations at the edge of the cells, which are associated with the impairments the wireless signal may suffer, such as path loss and fading. In order to estimate the QoS border cell that guarantees the QoS up to a given distance within the path, we use the Distance Reception Probability (DRP) module described in Section III-C1.

\section{B. Networking components}

1) Sensing the RANs module: This module is in charge of sensing the heterogeneous wireless Radio Access Networks 


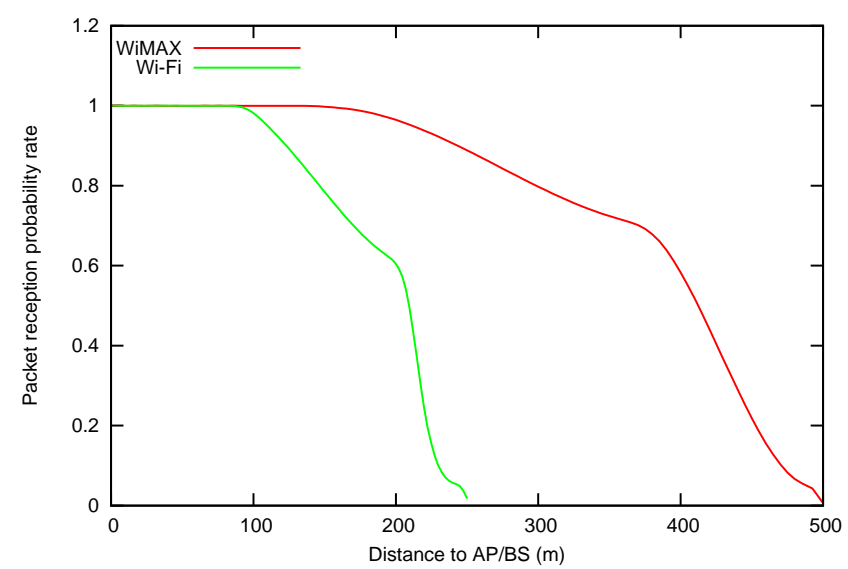

Fig. 4. Wi-Fi \& WiMAX Distance Reception Probability (DRP) model.

(RANs) available at the OBU. The module periodically sends and receives information about the network status, e.g., Router Advertisement (RA), Router Solicitation (RS), LINK SCAN. To interact with these interfaces, NAIRHA uses the IEEE 802.21 services, i.e., Media Independent Event Service (MIES) and Media Independent Command Service (MICS), to check the link status and received reports. When an event occurs at the PHY/MAC layer, the interfaces receive a trigger event that launches different sequential processes (decisionmaking, VHO execution); through the MIES, different events (e.g., LINK DETECTED, LINK DOWN or LINK RESPONSE) are notified to the upper layers in order to execute the different actions associated with a VHO process. Moreover, any further actions defined by the upper layers are executed by the lower layers using the primitives and commands provided by the MICS.

\section{Decision-making components}

At the decision-making process, several parameters are evaluated together in order to choose the best network candidate. Those parameters are the results of processes performed by the DRP module, the useful coverage estimation, and the application requirements and user preferences modules. We now proceed to describe these modules:

1) DRP module: NAIRHA not only considers the most suitable $\mathrm{CN}$ to switch to, but it also attempts to select the best time to leave the previous PoA and join the new one. To do so, the DRP module estimates the packet loss conditions associated with the different networks at different distances between the vehicle and the PoA. The estimation model used by this module should be chosen according to the characteristics of the underlying networks where it will be applied. Several models can be found in the literature [23], [24], [25]. Moreover, models can be calculated using the geolocation and the network status information measured by the vehicles, which it is stored in the MIIS database. For example, Figure 4 presents the packet loss as a function of distance to the PoA for both Wi-Fi and WiMAX technologies, obtained from our measurements of real Wi-Fi and WiMAX networks [26].

2) Useful coverage estimation module: Before describing the useful coverage estimation process, we must discuss the latency involved in a VHO process, since a high latency could be a symptom of packet loss and service disruption, thus downgrading the application performance; (2) describes the different components of this latency:

$$
\mathrm{VHOLat}=\mathrm{VHO}_{\mathrm{L} 2}+\mathrm{VHO}_{\mathrm{L} 3}+\mathrm{VHO}_{\mathrm{MIP}} \text {, }
$$

where $\mathrm{VHO}_{\mathrm{L} 2}$ is the latency referred to the association process at the link layer, while $\mathrm{VHO}_{\mathrm{L} 3}$ is related to the Internet Protocol (IP) level processes (i.e., IP address negotiation between the interface and the PoA). Finally, $\mathrm{VHO}_{\mathrm{MIP}}$ is the time taken by MIP for notifying the end-nodes and updating the home and foreign IP addresses when managing mobility.

The useful coverage estimation module has the task of calculating the minimum coverage time required to make it worthwhile to hand over to the candidate cell. Based on the UCT and the VHOLat, this module estimates the Cell Coverage Time (CCT) as:

$$
\mathrm{CCT}_{\text {min }}=\frac{\text { VHOLat }_{\text {max }}}{\alpha},
$$

where $\alpha$ is the proportion of the UCT during which the system is able to tolerate the adverse effects of VHO (which include both packet loss and latency).

Moreover, this module is also in charge of calculating the values of SP and $\mathrm{PW}$, such that a desirable SP must be smaller than the $\mathrm{CCT}_{\min }$, $\left(\mathrm{SP}_{\mathrm{des}}<\mathrm{CCT}_{\min }\right)$, meaning that, before the current neighborhood information becomes outdated upon reaching the CCT, the SP must obtain fresh information about the soon-to-be-reached neighborhood. This parameter determines how often the information must be collected.

We have also defined a desirable PW value, such that

$$
\mathrm{PW}_{\text {des }}=2 \cdot \beta \cdot \mathrm{CCT}_{\text {min }},
$$

where $\beta$ is a multiplier that can be tuned according to the OBU and the system performance, and it is expected to take values in the range of 1 to 2 (i.e., $1<\beta \leq 2$ ). Therefore, a suitable window size must be, at least, double the amount of SP time in terms of future information [27].

3) Application requirements and user preferences module: We have defined user profiles in order to classify the user preferences. Each profile considers both application requirements and the user's budget. Based on the Third Generation Partnership Project (3GPP) traffic classes and QoS specification [28], the defined profiles are:

- Maximum Performance: under this profile, the VHDA always selects the best performing network among all the possible choices, regardless of the associated cost.

- Streaming: the VHDA is optimized to choose those networks that offer not only high throughput, but also low packet loss ratio.

- Conversational: similarly to the streaming profile, this profile considers that having a low packet loss ratio as an important factor, but in this profile a low latency per packet is also critical when choosing a CN. Throughput is not so significant, and neither is cost.

- Minimum Cost: this profile is based on the user's budget, and it considers the price that user is willing to pay as 
the most important factor in the decision making process. If the user budget is low, the cheapest network available will be always the best choice.

The application requirements are a list of parameters that the VHDA takes into account, in conjunction with the user preferences, for evaluating the best $\mathrm{CN}$. This list contains $\mathrm{N}$ parameters evaluated by a Multiple Criteria Decision-Making (MCDM) algorithm. That way, all of them are considered and weighted by the decision-making process when selecting the most suitable network. Parameters include:

- Throughput: the minimum throughput required by the application.

- Latency per packet: the average latency that the application is able to tolerate.

- Packet loss ratio: the losses that the application can tolerate.

- Price per MB: the price that the user is willing to pay for the service.

4) SAW-based network assessment function: As we mentioned before, an MCDM algorithm is used to evaluate the criteria enumerated above. The algorithm is based on the SAW algorithm. We define the ratio among the Candidate Network Parameters (CNP) and the Application Requirement Parameters (ARP), called the Parameter Ratio (PR), as:

$\mathrm{PR}_{i}= \begin{cases}\frac{\mathrm{ARP}_{\mathrm{i}}}{\mathrm{CNP}_{\mathrm{i}}} & \text { if } \mathrm{i} \in\{\text { PriceMb,PacketLatency,PacketLoss }\} \\ \frac{\mathrm{CNP}_{\mathrm{i}}}{\mathrm{ARP}} & \text { if } \mathrm{i}=\text { Throughput, }\end{cases}$

assuming $\mathrm{ARP}_{\mathrm{i}}$, and $\mathrm{CNP}_{\mathrm{i}}$ are greater than zero. In order to adjust the importance (relative weight) of the requirements for each $\mathrm{CN}$, as a function of the user profile, a multiplier is required. Factors $\omega_{\mathrm{i}}$ are profile-specific, and allow modifying the weight of each PR element, according to:

$$
\mathrm{CN}_{\text {MCDMValue }}=\sum_{\mathrm{i}=1}^{\mathrm{N}} \omega_{\mathrm{i}} \mathrm{PR}_{\mathrm{i}},
$$

where $\mathrm{i}$ is an element of the application requirements list.

The weigths need to be callibrated properly; they can be calculated in real time at the MIIS server based on the information gathered from the vehicles, and also from any other network entity, as long as such functionality relies on the MIIS service of the IEEE 802.21 standard; such information includes the geolocation, availability and state of the network, and whether or not the vehicle had just performed a handover. That collected data provides the information needed to calculate the weights in a centralized manner but the decision-making process is performed at the vehicle's OBU, distributed in the sense that vehicles will make the decision based upon their own profiles and requirements, as the distrubuted solution applied by Fazio et al. [29].

To obtain the results presented in this paper, we used a Monte Carlo process whose details are included in Section IV-C.
5) Decision-making process: For the decision-making process, NAIRHA evaluates three factors in the following priority order:

- The Useful Coverage Time (UCT) is calculated and evaluated to decide, based on this value, whether it is worth handing over to the evaluated Candidate Network $(\mathrm{CN})$; networks with a too short UCT are disregarded.

- Based on the Distance Reception Probability (DRP) required, NAIRHA verifies whether the Candidate Network $(\mathrm{CN})$ is able to fulfill such requirements. Networks with a DRP lower than the minimum required are not selected.

- Finally, the Simple Additive Weighting (SAW) function calculates the $\mathrm{CN}_{\mathrm{MCDMV}}$ alue as shown in (6) for each Candidate Network $(\mathrm{CN})$. Once all the values are obtained, they are compared, and the $\mathrm{CN}$ with the highest value is chosen. It means that the chosen network is the most suitable network when attempting to fulfill the application requirements under a certain user profile.

When the decision-making process finishes, the VHO execution process performs its tasks and seamlessly switches from the old network to the selected $\mathrm{CN}$, executing the MIP notification process and redirecting the traffic flows.

The pseudo code in algorithms III.1 (procedures) and III.2 (main) describes the auxiliary procedures and the main procedure, respectively, that rule NAIRHA.

6) Discussion: In the previous subsection we have introduced the decision making process in NAIRHA, which is a three-step process. In the first two steps we exclude the infeasible CNs, and then in the third step we select the best $\mathrm{CN}$ based on the requirements, as described by the auxiliary and main procedures in the pseudo-code presented.

Another approach is to formulate this decision-making process as four distinct single-objective constrained optimization problems. In each of these problems the objective is either throughput, latency per packet, packet loss ratio, or price, and the remaining objectives become the constrains of that optimization problem. For example in the throughput maximization problem, the constrains are the latency per packet, packet loss ratio, and the price. Moreover, the optimization problem must include other constraints on UCT and DRP (mentioned in the first and second steps of NAIRHA's decision process). However, the boundaries of these requirements are not clear and the training is performed from a subjective point of view, which makes the definition of the exact constrains extremely difficult. To overcome these difficulties, and to propose a practical solution, we define the multi-criteria decision making procedure and determine the weights for each profile using a Monte Carlo process.

\section{Algorithm Evaluation}

This section describes the tools, the scenario, and the tuning of the NAIRHA parameters used to evaluate the performance of our proposed algorithm.

\section{A. Simulation tools}

The National Institute of Standards and Technology (NIST) mobility package for the Network Simulator (ns-2) [30], 


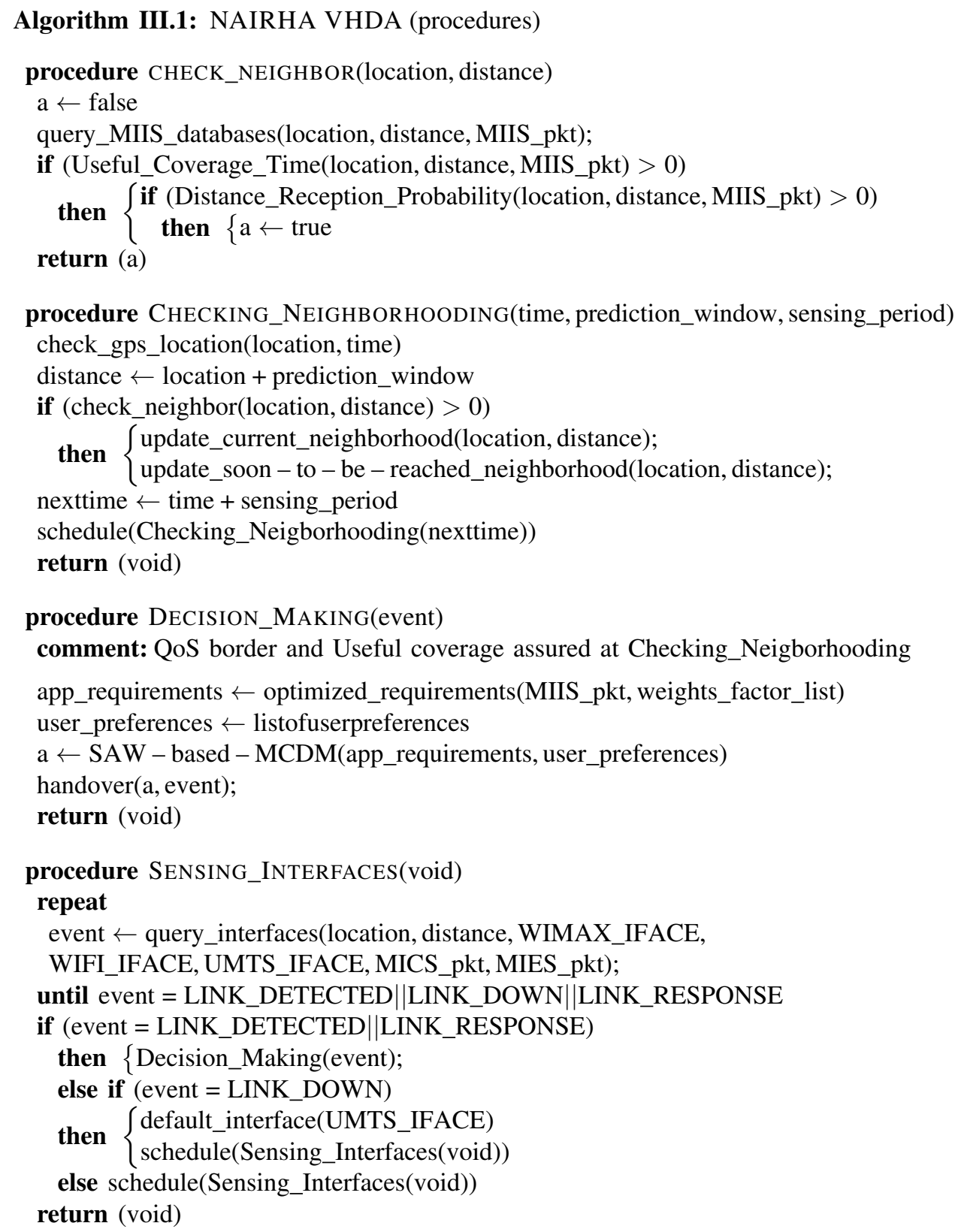

\section{Algorithm III.2: NAIRHA VHDA (main)}

\section{main}

while 1
do $\left\{\begin{array}{l}\text { comment: keeping up-to-date the neighborhoods } \\ \text { Checking_Neighborhooding(now, prediction_window, sensing_period) } \\ \text { comment: Sensing the ifaces and triggering the decision-making process } \\ \text { Sensing_Interfaces() }\end{array}\right.$ 
TABLE I

VHO SCHEME COMPONENTS.

\begin{tabular}{llll}
\hline Component & Wi-Fi & WiMAX & UMTS \\
\hline Access Point & 8 & 3 & 1 \\
Nominal data rate (Mbps) & 54 & 70 & 5 \\
Data rate offered (Mbps) & 28.2 & 16.3 & 2.7 \\
VHO latency (ms) [35], [36] & 1080 & 2665 & - \\
Advertisement interval (ms) & 100 & 5000 & - \\
Coverage (m) & 500 & 1000 & 5000 \\
\hline
\end{tabular}

[31], in conjunction with EURANE [32], can simulate Wi-Fi, WiMAX, and UMTS technologies, including VHO. Furthermore, the NIST add-on also enables the MIES and the MICS of the IEEE 802.21 standard to interact with heterogeneous network interfaces under homogeneous standard primitives.

Since NAIRHA requires the IEEE 802.21 Media Independent Information Service (MIIS), we have developed (by extending the NIST add-on) an MIIS considering local and remote databases which store the PoA container information, being able to read and write information via XML files, strictly following the IEEE 802.21 standard. Our implementation is also capable of updating the status of the PoA container via notifications performed by the vehicles, as suggested by Andrei et al. in [33].

We have also implemented a Global Positioning System (GPS) add-on module for ns- 2 which manages the GPS coordinates, maps, and routes, to select an itinerary to travel from the current geolocation to any destination. The GPS module also translates the geolocation coordinates into traveling time, in order to allow the NAIRHA algorithm to know where the vehicle is expected to be at any moment in the future.

In order to be able to simulate and to study the impact of the DRP, we have modified the Medium Access Control (MAC) layer behaviour of both 802.11 and 802.16 protocols in the simulator. All the modifications incorporate the prior modifications done by the NIST at the MAC layer [34].

\section{B. Simulation scheme}

In our experiments we used a scenario with vehicles moving at $32 \mathrm{~km} / \mathrm{h}$ from the Universitat de València campus (source) to the Universitat Politècnica de València campus (destination) in the city of Valencia, Spain. Figure 5 shows an itinerary covering a distance of $5.5 \mathrm{~km}$ in a $3.75 \mathrm{~km}^{2}$ area. Our GPS module manages all the coordinates for the itinerary. Moreover, the MIIS provides information about the available networks and their respective PoAs within the simulated area, as also shown in Figure 5. Table I summarizes the main configuration set for the experiments. As observed, there are 1 UMTS, $8 \mathrm{Wi}-\mathrm{Fi}$, and $3 \mathrm{WiMAX}$ PoAs covering different areas with distinct bandwidth capacity. It is important to point out that UMTS covers the whole scenario, meaning that the UMTS technology is always the backup connectivity technology for this set of experiments.

Moreover, we have configured each network in the scenario with different performance parameters. By doing this, we generate different alternatives to evaluate the CNs. Table II presents the parameter set for each network, and Table III

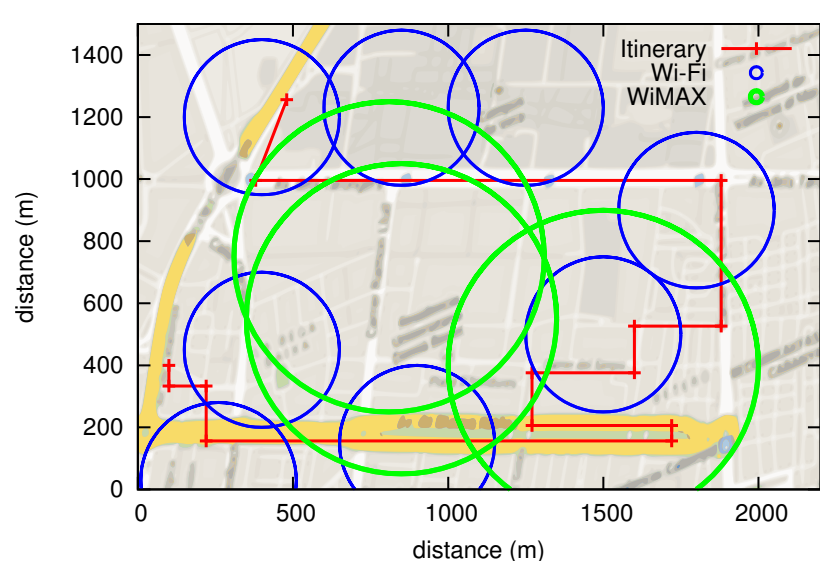

Fig. 5. Coverage scenario

TABLE III

APPLICATION REQUIREMENTS FOR A VIDEO ON DEMAND SESSION.

\begin{tabular}{ll}
\hline Parameter & Value \\
\hline Price per MB & 0.05 \\
Latency per packet & 100 \\
Packet loss ratio & 2 \\
Throughput (Mbps) & 1 \\
\hline
\end{tabular}

presents the minimum requirements for the video session that must be fulfilled by the chosen networks during the simulation. We have considered video streaming traffic since video is expected to be a major component of the increase in demand for mobile services in the near future.

\section{Tuning NAIRHA parameters}

The VHOLat considered for each technology has been extracted from real measurements of Wi-Fi handovers performed at the Universitat Politècnica de València campus, while the WiMAX handovers have been performed at the Universidad de Murcia campus; these measurements are consistent with the ones presented by Tsao et al. and Yoo et al. [35], [36]. We have set $\alpha$ to $5 \%$, and $\beta$ is 1 .

The Distance Reception Probability (DRP) used for these experiments is based on real measurements. To obtain a valid model for the channel behavior, we have performed several measurements within the Universitat Politècnica de València campus and the University of Murcia campus, obtaining Wi-Fi and WiMAX results, respectively. For measurement purposes, a 1500-byte packet size was used. It is important to point out that the measurements were taken at the MAC level, to model the PHY/MAC behavior. (7) and (8) present the reception probability as functions of distance based on a curve fitting interpolation for the performance of the two networks mentioned above. The threshold chosen for the DRP was $40 \%$.

Regarding the calibration of the weight values $\omega_{i}$, to calculate the appropriate values of each parameter, for the different user profiles, we have adopted a 2-step Monte Carlo process. The Monte Carlo process is fed by a training set, considering 10 different networks with distinct performance and characteristics (among Wi-Fi, WiMAX, and UMTS). We defined a 
TABLE II

NETWORK PARAMETERS.

\begin{tabular}{|c|c|c|c|c|c|c|}
\hline & Technology & $\begin{array}{l}\text { Price } \\
\text { MB }\end{array}$ & per & Latency per Packet & Packet Loss Ratio & Throughput (Mbps) \\
\hline PoA-1 & UMTS & 0.9 & & 25.55 & 0.76 & 1.41 \\
\hline PoA-2 & Wi-Fi & 0.8 & & 15.22 & 1.19 & 1.44 \\
\hline PoA-3 & Wi-Fi & 0.4 & & 30.44 & 2.38 & 0.72 \\
\hline PoA-4 & WiMAX & 0.15 & & 17.54 & 2.74 & 1.18 \\
\hline PoA-5 & $\mathrm{Wi}-\mathrm{Fi}$ & 0.0513 & & 23.7432 & 1.8564 & 0.931 \\
\hline PoA-6 & WiMAX & 0.02 & & 60.88 & 4.76 & 0.36 \\
\hline PoA-7 & Wi-Fi & 0.075 & & 35.08 & 3.1510 & 0.59 \\
\hline PoA-8 & Wi-Fi & 1.2 & & 0.55 & 0.86 & 1.81 \\
\hline PoA-9 & Wi-Fi & 0.8 & & 0.75 & 0.98 & 1.69 \\
\hline PoA-10 & WiMAX & 0.0375 & & 70.16 & 3.5606 & 0.2950 \\
\hline PoA-11 & Wi-Fi & 0.7692 & & 0.858 & 1.3416 & 1.1603 \\
\hline PoA-12 & Wi-Fi & 0.5128 & & 1.17 & 1.5288 & 1.0833 \\
\hline
\end{tabular}

$$
\begin{gathered}
\mathrm{DRP}_{\mathrm{Wi}-\mathrm{Fi}}= \begin{cases}1 & \text { if } \mathrm{d} \leq 100 \\
0.571+0.0138 \cdot \mathrm{d}-0.00012 \cdot \mathrm{d}^{2}+2.912 \mathrm{e}^{-07} \cdot \mathrm{d}^{3} & \text { if } 100<\mathrm{d} \leq 210 \\
165.489-2.0342 \cdot \mathrm{d}+0.00833 \cdot \mathrm{d}^{2}-1.139 \mathrm{e}^{-05} \cdot \mathrm{d}^{3} & \text { if } 210<\mathrm{d} \leq 250 \\
0 & \text { if } \mathrm{d}>250\end{cases} \\
\mathrm{DRP}_{\text {WiMAX }}= \begin{cases}1 & \text { if } \mathrm{d} \leq 150 \\
0.4889+0.00765 \cdot \mathrm{d}-3.485 \mathrm{e}^{-05} \cdot \mathrm{d}^{2}+4.258 \mathrm{e}^{-08} \cdot \mathrm{d}^{3} & \text { if } 150<\mathrm{d} \leq 375 \\
-44.908+0.333 \cdot \mathrm{d}-0.000798 \cdot \mathrm{d}^{2}+6.222 \mathrm{e}^{-05} \cdot \mathrm{d}^{3} & \text { if } 375<\mathrm{d} \leq 500 \\
0 & \text { if } \mathrm{d}>500\end{cases}
\end{gathered}
$$

training set with a total of $270 \mathrm{VHO}$ decisions, combining the different networks at different utilization states. The decisions in the training set were made from a subjective point of view, considering all the Application Requirement Parameters (ARP) and the Candidate Network Parameters (CNP).

The first step of the Monte Carlo process was to determine the best $\omega$ values, out of three million runs, based on the training set. The success rate is measured in terms of similarity to the decisions used as input to the process. Once the $\omega_{\mathrm{i}}$ values were chosen, we proceeded to the second step of the Monte Carlo process, adding other three million runs to refine the $\omega_{i}$ values obtained in the first step. In particular, the variation interval for the $\omega_{\mathrm{i}}$ values was $1 \%$. This second step provides even more accurate $\omega$ values. Table IV presents the $\omega$ values optimized for each user profile. These sets of values achieve a success ratio of about $82 \%$ for the VHO decision process when NAIRHA is applied.

\section{Performance evaluation}

To evaluate the NAIRHA performance we have performed numerous simulations varying the user profile. We have compared the performance of NAIRHA to the performance of three other algorithms available in the literature. Therefore, we have also implemented and performed the Techaware [37], Multi-ACcess network Handover algorithm for vehicUlar environments (MACHU) [27] and the Geolocationbased Multi-ACcess network Handover algorithm for vehicUlar environments (Geo-MACHU) [26] VHDAs in order to conduct experiments under the same conditions and to perform a fair comparison. A 95\% confidence interval was obtained for all the simulations performed for all algorithms.

Figure 6 depicts the connectivity adopted by NAIRHA for each user profile, for the same ARP. As can observed, different networks are chosen depending on the selected profile. In order to compare the performance levels associated with the different algorithms, Figure 7 presents the connectivity behaviour of the Geo-MACHU algorithm with a DRP threshold of $40 \%$ at the QoS border, showing the active network interfaces, and performing 11 VHO events, as well as the performance of Tech-aware and MACHU, performing 18 and 15 VHO events, respectively. Table $\mathrm{V}$ summarizes the connectivity behaviour by presenting the number of $\mathrm{VHO}$ events. As shown, a different number of events take place depending on the user profile. Despite the conversational and maximum performance profiles having resulted in the same number of VHO events, the selected networks are different, thus reaching different performance. To reinforce this profile dependency, Figure 8 presents the dwell time per technology, that is, the total time each interface was active during the simulation.

With respect to cost, we can observe in Figure 9 that the different user profiles are also associated with different costs. We can confirm that the minimum cost profile was able to meet the original goal by choosing the networks in an accurate manner, thereby reducing the total cost of the video session. However, this profile is intended to optimize the cost in detriment of the remaining application requirements. In fact, we can observe in Figures 10, 11, and 12 that the minimum cost profile achieves poor performance, having a packet delivery 
TABLE IV

$\omega$ VALUES OPTIMIZED FOR THE USER PROFILES.

\begin{tabular}{lllll}
\hline ARP & Minimum Cost & Streaming & Conversational & Maximum Performance \\
\hline PriceMB & 0.4637620 & 0.4218970 & 0.2578700 & 0.0469420 \\
PacketLatency & 0.1068350 & 0.4216220 & 0.1638400 & 0.0617170 \\
PacketLoss & 0.0339010 & 0.1348730 & 0.2269100 & 0.3986950 \\
Throughput & 0.3955020 & 0.0216080 & 0.3513700 & 0.4926470 \\
\hline
\end{tabular}

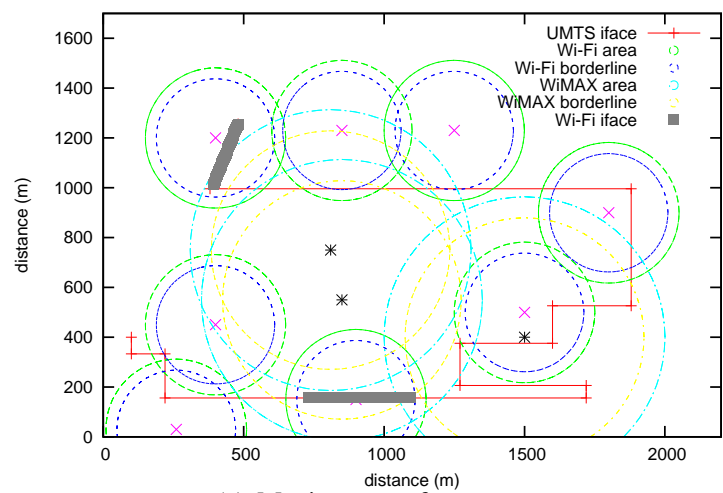

(a) Maximum performance.

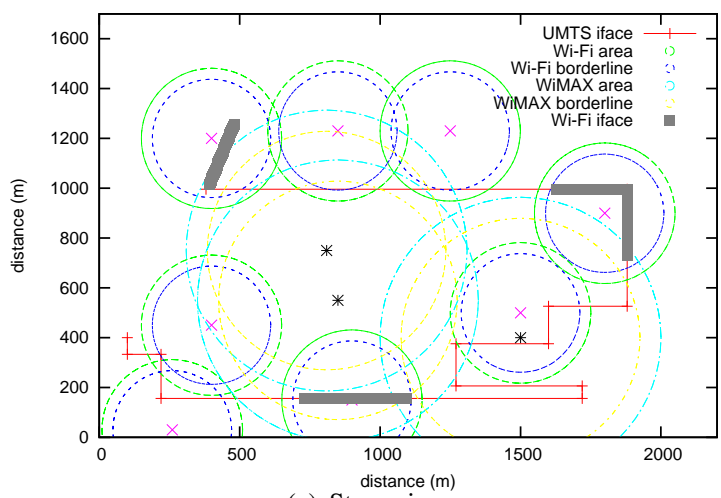

(c) Streaming.

Fig. 6. Video demand connectivity profile comparison.

TABLE V

VHO EVENTS

\begin{tabular}{ll}
\hline & VHO events \\
\hline Tech-Aware & 18 \\
MACHU & 15 \\
Geo-MACHU 40\% & 11 \\
NAIRHA Maximum performance & 5 \\
NAIRHA Conversational & 5 \\
NAIRHA Streaming & 3 \\
NAIRHA Minimum Cost & 9 \\
\hline
\end{tabular}

ratio of only $65 \%$; the same is true for Geo-MACHU, and even worse performance is achieved by the MACHU and Tech-aware solutions. We also observe that the maximum performance profile achieves the highest performance (i.e., low latency, high throughput, and low packet loss rate) by selecting the CNs with better performance, but paying the highest cost for those high quality services.

Figure 10 presents the throughput achieved by each user

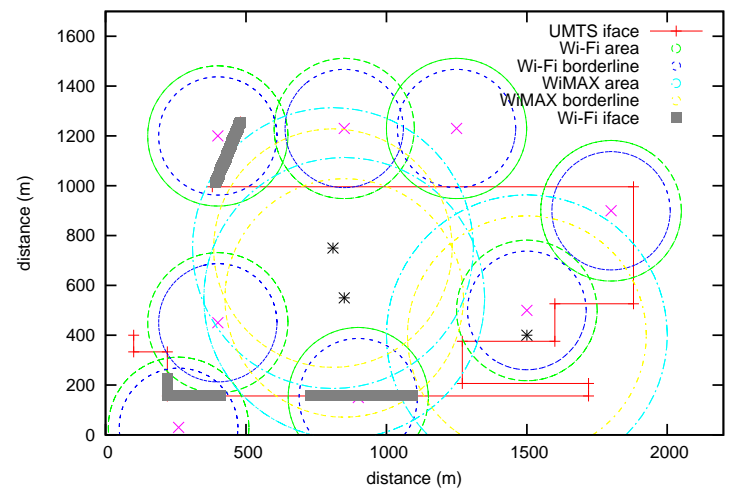

(b) Conversational.

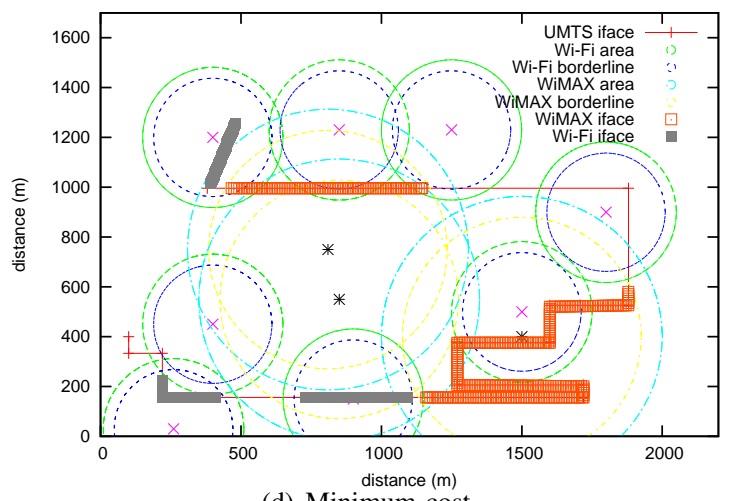

(d) Minimum cost

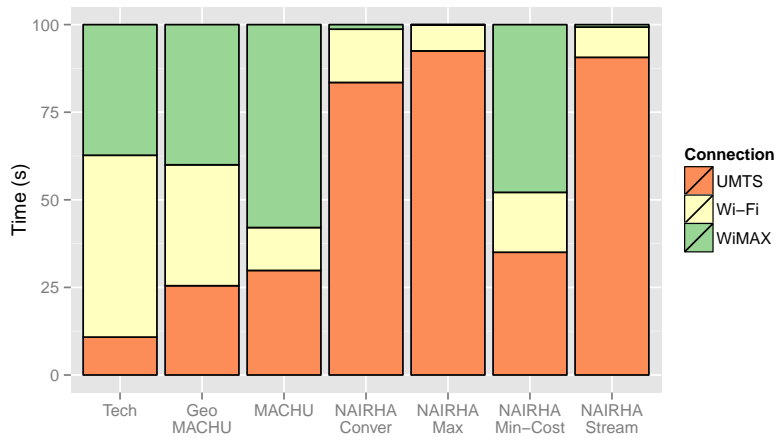

Fig. 8. Dwell time comparison.

profile under NAIRHA and the other VHDAs. We can observe that the streaming, conversational and maximum performance profiles obtain the $1 \mathrm{Mbps}$ desired, while the Minimum Cost and Geo-MACHU $40 \%$ profiles achieve about $640 \mathrm{Kbps}$; the 


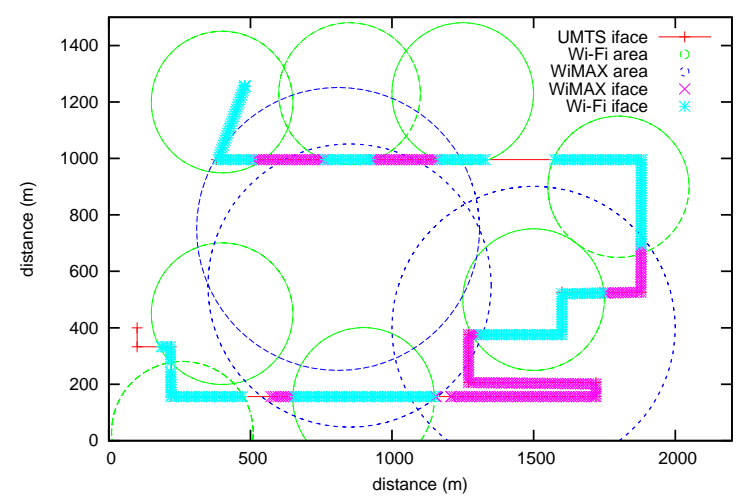

(a) Tech-aware.

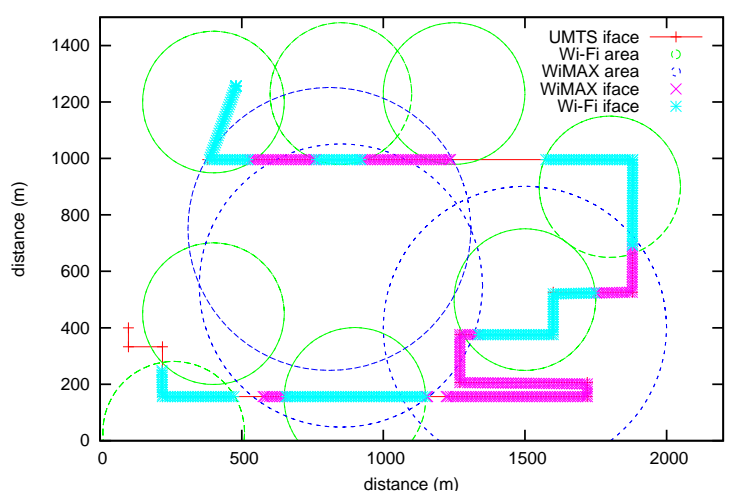

(b) MACHU.

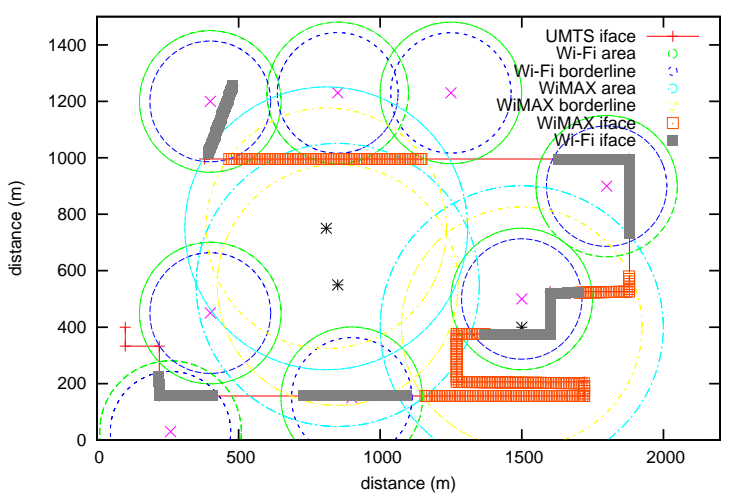

(c) Geo-MACHU $(40 \%)$.

Fig. 7. Other VHA connectivity comparison.

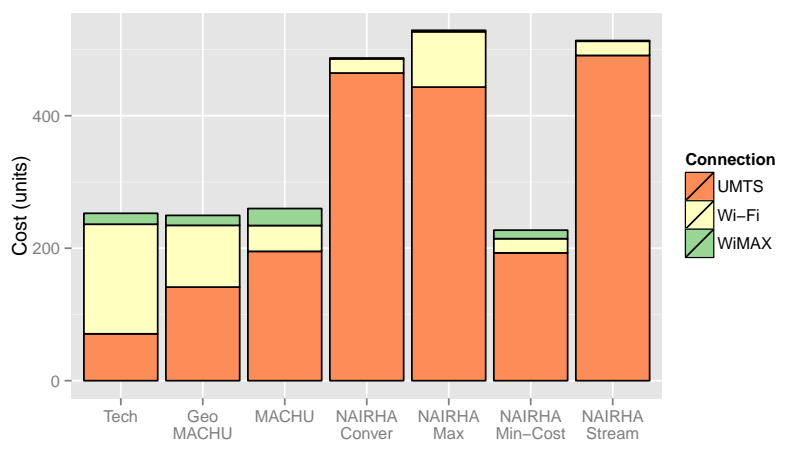

Fig. 9. Cost comparison.

MACHU and Tech-Aware solutions are hardly able to surpass the $500 \mathrm{Kbps}$ threshold, since their priority is not performance, but rather the cost, geolocation or prioritized technology. Concerning latency and packet loss, we observe that streaming, conversational, and maximum performance profiles achieve different trade-offs between performance and cost. We can observe that those profiles achieve better performance in terms of latency (Figure 11) and packet losses (Figure 12), while the other profiles do not optimize these parameters.

\section{CONCLUSION}

In this paper we have proposed a Vertical Handover Decision Algorithm (VHDA) called NAIRHA. The algorithm

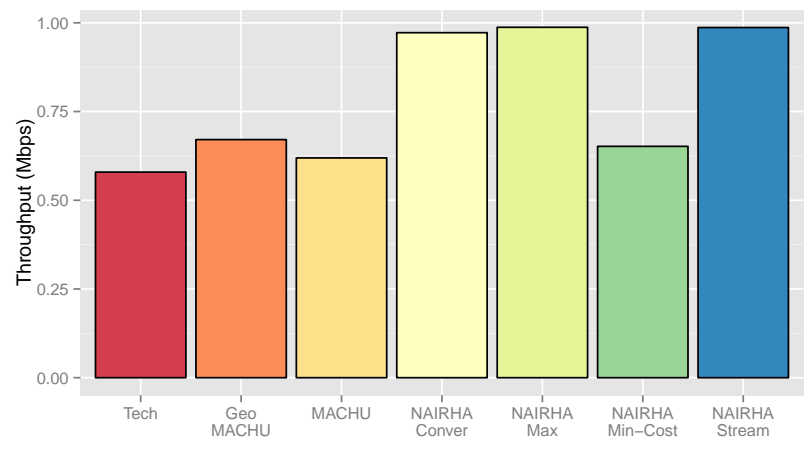

Fig. 10. Throughput comparison.

selects the most suitable candidate network that fulfils the connectivity requirements, taking into account the user preferences, within vehicular contexts. To do so, NAIRHA takes advantage of the current features of the OBUs such as GPS-based geolocation and geonavigation, multiple wireless network interfaces, continuous power supply, and powerful computing resources. Moreover, the services provided by the IEEE 802.21 standard help to empower NAIRHA.

Throughout simulation we have demonstrated that NAIRHA is able to accurately select the best candidate network according to the connectivity requirements based on the user preferences and application requirements.

Our approach targets infrastructure-based vehicular net- 


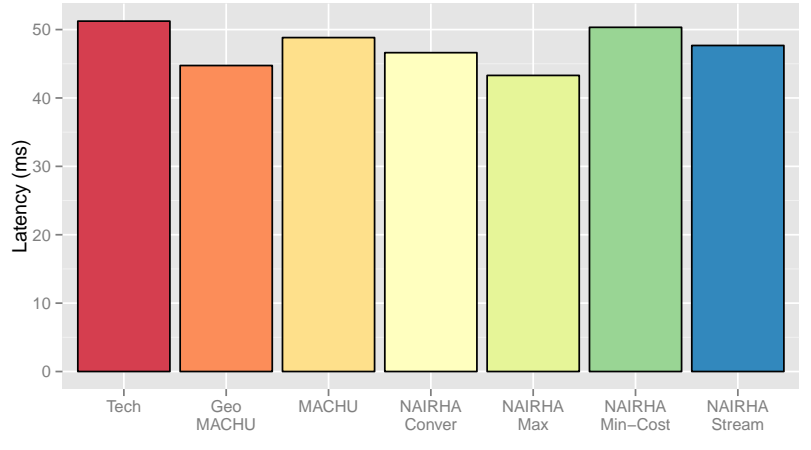

Fig. 11. Latency comparison.

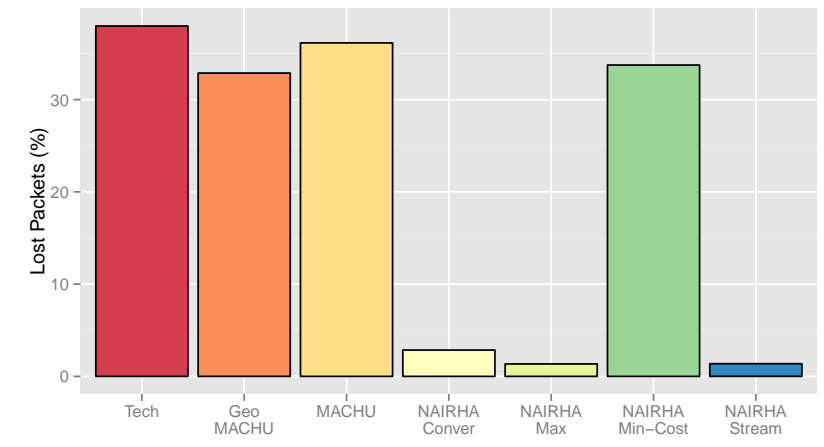

Fig. 12. Packet loss comparison.

works rather than VANET-based solutions. Moreover, our approach relies on and benefits from the IEEE 802.21 standard. A future improvement will consist on including VANET technologies and protocols (e.g., DSRC and IEEE 802.11p) to aggregate the information collected through car-to-car communications, and to deliver such information to the MIIS databases to enhance the knowledge and the decisions adopted under our approach.

\section{ACKNOWLEDGMENT}

This work was partially supported by the Ministerio de Economía y Competitividad, Spain, under Grants TIN201127543-C03-01 and BES-2012-052673, and by the Universitat Politècnica de València through the ABATIS project (PAID05-12).

We also acknowledge support from the Science Foundation Ireland under Grants No. 10/IN.1/I3007 and 10/CE/I1853.

\section{REFERENCES}

[1] I. Broustis and M. Faloutsos, "Routing in Vehicular Networks: Feasibility, Modeling, and Security," International Journal of Vehicular Technology, 2008. [Online]. Available: http://www.hindawi.com/GetArticle.aspx?doi= $10.1155 / 2008 / 267513 \& \# 38 ; \mathrm{e}=\mathrm{cta}$

[2] "IEEE standard for local and metropolitan area networks- part 21: Media independent handover," Tech. Rep., 2009. [Online]. Available: http://dx.doi.org/10.1109/ieeestd.2009.4769367

[3] M. Stemm and R. H. Katz, "Vertical handoffs in wireless overlay networks," Springer Mobile Networks and Applications., vol. 3, no. 4, pp. 335-350, 1998. [Online]. Available: http://dx.doi.org/10.1023/A: 1019197320544
[4] J. Marquez-Barja, C. T. Calafate, J.-C. Cano, and P. Manzoni, "An overview of vertical handover techniques: Algorithms, protocols and tools," Computer Communications, vol. 34, no. 8, pp. 985-997, Jun. 2011. [Online]. Available: http://dx.doi.org/10.1016/j.comcom.2010.11.010

[5] A. Dutta, S. Madhani, W. Chen, O. Altintas, and S. Cai, "GPS-IP based Fast-hanoff for Mobiles," in 3rd New York Metro Area Networking Workshop, Sep. 2003. [Online]. Available: http: //www.cs.columbia.edu/dutta/research/gps-ip-nyman-final.pdf

[6] J. Montavont and T. Noel, "IEEE 802.11 Handovers Assisted by GPS Information," in IEEE International Conference on Wireless and Mobile Computing, Networking and Communications., 2006, pp. 166-172. [Online]. Available: http://dx.doi.org/10.1109/wimob.2006.1696358

[7] W. D. Hsiao, Y. X. Liu, and H. C. Chao, "An intelligent WiMAX mobile network handoff mechanism with GPS consideration," in International ACM Conference on Mobile Technology, Applications, and Systems, 2008. [Online]. Available: http://dx.doi.org/10.1145/1506270.1506404

[8] M. Ylianttila, J. Makela, and K. Pahlavan, "Analysis of handoff in a location-aware vertical multi-access network," Elsevier Computer Networks, vol. 47, no. 2, pp. 185-201, Feb. 2005. [Online]. Available: http://www.sciencedirect.com/science/article/pii/S138912860400194X

[9] C. Gu, M. Song, Y. Zhang, and J. Song, "Access network selection strategy using position prediction in heterogeneous wireless networks," Frontiers of Electrical and Electronic Engineering in China, vol. 5, no. 1, pp. 23-28, Mar. 2010. [Online]. Available: http://dx.doi.org/10.1007/s11460-009-0064-4

[10] H. Hamad, R. Salama, and S. Elkourd, "A Location Aided Fast Handoff Protocol for the Next Generation Wireless Systems," in International Conference on Communications in Computing, Jul. 2008. [Online]. Available: http://site.iugaza.edu.ps/hhamad/files/2010/03/LAFHP.pdf

[11] S. Wang, C. Fan, C. H. Hsu, Q. Sun, and F. Yang, "A Vertical Handoff Method via Self-Selection Decision Tree for Internet of Vehicles," IEEE Systems Journal. [Online]. Available: http://dx.doi.org/10.1109/jsyst.2014.2306210

[12] L.-C. Wang, A. Chen, and A. Chen, "Network Selection with Joint Vertical and Horizontal Handoff in Heterogeneous WLAN and Mobile WiMax Systems," in IEEE 65th Vehicular Technology Conference. IEEE, Apr. 2007, pp. 794-798. [Online]. Available: http://dx.doi.org/10.1109/vetecs.2007.173

[13] B. Ma and X. Liao, "Speed-adaptive vertical handoff algorithm based on fuzzy logic in vehicular heterogeneous networks," in 9th International Conference on Fuzzy Systems and Knowledge Discovery. IEEE, May 2012, pp. 371-375. [Online]. Available: http://dx.doi.org/10.1109/fskd.2012.6234358

[14] Y. Tao and R. Peng, "A fuzzy logic vertical handoff algorithm with motion trend decision," in 6th IEEE International Forum on Strategic Technology, Aug. 2011, pp. 1280-1283. [Online]. Available: http://dx.doi.org/10.1109/ifost.2011.6021253

[15] J. D. Martinez-Morales, U. Pineda-Rico, and E. Stevens-Navarro, "Performance comparison between MADM algorithms for vertical handoff in 4G networks," in 7th International IEEE Conference on Electrical Engineering Computing Science and Automatic Control (CCE), Sep. 2010, pp. 309-314. [Online]. Available: http://dx.doi.org/10.1109/iceee.2010.5608646

[16] A. Ismail and B.-h. Roh, "Adaptive Handovers in heterogeneous networks using fuzzy MADM," in IEEE International Conference on Mobile IT Convergence (ICMIC), Sep. 2011, pp. 99-104.

[17] K. Shafiee, A. Attar, and V. C. M. Leung, "Optimal Distributed Vertical Handoff Strategies in Vehicular Heterogeneous Networks," Selected Areas in Communications, IEEE Journal on, vol. 29, no. 3, pp. 534-544, Mar. 2011. [Online]. Available: http://dx.doi.org/10.1109/jsac.2011.110304

[18] A. De La Oliva, L. Eznarriaga, C. J. Bernardos, P. Serrano, and A. Vidal, "IEEE 802.21: A shift in the Media Independence," in IEEE Future Network \& Mobile Summit, Jun. 2011, pp. 1-8.

[19] K. Inoyatov, S. Tursunova, I. Kim, and Y.-T. Kim, "IEEE 802.21 MIH in Linux Kernel space for cognitive and smart handovers," in 12th IFIP/IEEE International Symposium on Integrated Network Management (IM 2011) and Workshops, May 2011, pp. 674-677. [Online]. Available: http://dx.doi.org/10.1109/inm.2011.5990653

[20] P. Soderman, K.-J. Grinnemo, G. Cheimonidis, Y. Ismailov, and A. Brunstrom, "An SCTP-based Mobility Management Framework for Smartphones and Tablets," in 26th IEEE International Conference on Advanced Information Networking and Applications Workshops, Mar. 2012, pp. 1107-1112. [Online]. Available: http://dx.doi.org/10.1109/waina.2012.84 
[21] G. Albertengo, W. Buttazzo, A. Tragno, M. Ricca, A. Bragagnini, and R. Quasso, "Smartphone enabled connected vehicles pave the way to intelligent mobility," in World Telecommunications Congress 2014, Jun. 2014, pp. 1-6. [Online]. Available: http: //ieeexplore.ieee.org/xpls/abs_all.jsp?arnumber $=6840022$

[22] N. A. M. Nordin, Z. A. Zaharudin, M. A. Maasar, and N. A. Nordin, "Finding shortest path of the ambulance routing: Interface of A algorithm using C\# programming," in IEEE Symposium on Humanities, Science and Engineering Research (SHUSER), Jun. 2012, pp. 1569-1573. [Online]. Available: http://dx.doi.org/10.1109/shuser.2012.6268841

[23] R. Samano-Robles and A. Gameiro, "A packet reception model for cooperative relaying diversity in wireless multicell networks," in IEEE International Conference on Consumer Electronics (ICCE), Sep. 2011, pp. 327-331. [Online]. Available: http://dx.doi.org/10.1109/icce-berlin.2011.6031885

[24] W. L. Tan, P. Hu, and M. Portmann, "Experimental evaluation of measurement-based SINR interference models," in IEEE International Symposium on a World of Wireless, Mobile and Multimedia Networks (WoWMoM), Jun. 2012, pp. 1-9. [Online]. Available: http://dx.doi.org/10.1109/wowmom.2012.6263695

[25] J. Gozalvez, M. Sepulcre, and R. Bauza, "IEEE 802.11p vehicle to infrastructure communications in urban environments," IEEE Communications Magazine, vol. 50, no. 5, pp. 176-183, May 2012 [Online]. Available: http://dx.doi.org/10.1109/mcom.2012.6194400

[26] J. Marquez-Barja, C. T. Calafate, J.-C. Cano, and P. Manzoni, "A geolocation-based vertical handover decision algorithm for vehicular networks," in IEEE 37th Conference on Local Computer Networks (LCN), Oct. 2012, pp. 360-367. [Online]. Available: http://dx.doi.org/10.1109/lcn.2012.6423648

[27] - "MACHU: A novel vertical handover algorithm for vehicular environments," in IEEE Wireless Telecommunications Symposium (WTS 2012), Apr. 2012. [Online]. Available: http://dx.doi.org/10.1109/WTS.2012.6266087

[28] 3GPP and ETSI, "Digital cellular telecommunications System (Phase 2+); Universal Mobile Telecommunications System (UMTS); LTE; Quality of Service (QoS) concept and architecture(3GPP TS 23.107 version 11.0.0 Release 11)," Tech. Rep., 2012. [Online]. Available: http://www.etsi.org/deliver/etsi_ts/123100_ 123199/123107/11.00.00_60/ts_123107v110000p.pdf

[29] P. Fazio, F. De Rango, C. Sottile, and A. F. Santamaria, "Routing Optimization in Vehicular Networks: A New Approach Based on Multiobjective Metrics and Minimum Spanning Tree," International Journal of Distributed Sensor Networks, vol. 2013, pp. 1-13, 2013. [Online]. Available: http://dx.doi.org/10.1155/2013/598675

[30] K. Fall and K. Varadhan, "ns Notes and Documents." The VINT Project. UC Berkeley, LBL, USC/ISI, and Xerox PARC, Jun. 2009. [Online]. Available: http://www.isi.edu/nsnam/ns/ns-documentation.html

[31] Advanced Network Technology Division- National Institute of Standards and Technology, "Seamless and Secure Mobility," http://www.antd.nist.gov/seamlessandsecure/.

[32] B. V. Ericsson Telecommunicatie, "EURANE - enhanced UMTS radio access network extensions for ns-2." [Online]. Available: http://eurane.ti-wmc.nl/eurane/

[33] V. Andrei, E. C. Popovici, O. Fratu, and S. V. Halunga, "Development of an IEEE 802.21 Media Independent Information Service," in IEEE International Conference on Automation Quality and Testing Robotics (AQTR), May 2010, pp. 1-6. [Online]. Available: http://dx.doi.org/10.1109/aqtr.2010.5520819

[34] Advanced Network Technology Division- National Institute of Standards and Technology, "The network simulator ns-2 NIST add-on MAC 802.11," http://www.antd.nist.gov/seamlessandsecure. [Online]. Available: http: //www.antd.nist.gov/seamlessandsecure/files/mobility/doc/MAC802 11.pdf

[35] S.-L. Tsao, Y.-L. Chen, and C.-H. Chang, "Evaluation of Scan and Association Process for Real-Time Communication in Mobile WiMAX," IEEE Transactions on Wireless Communications, vol. 9, no. 11, pp. 3320-3323, Nov. 2010. [Online]. Available: http: //dx.doi.org/10.1109/twc.2010.091510.090619

[36] S.-J. Yoo, D. Cypher, and N. Golmie, "Predictive link trigger mechanism for seamless handovers in heterogeneous wireless networks," Wirel. Commun. Mob. Comput., vol. 9, no. 5, pp. 685-703, 2009. [Online]. Available: http://dx.doi.org/10.1002/wcm.620

[37] J. Marquez-Barja, C. T. Calafate, J.-C. Cano, and P. Manzoni, "Evaluation of a technology-aware vertical handover algorithm based on the IEEE 802.21 standard," in IEEE Wireless Communications and
Networking Conference (WCNC), Mar. 2011, pp. 617-622. [Online] Available: http://dx.doi.org/10.1109/WCNC.2011.5779204

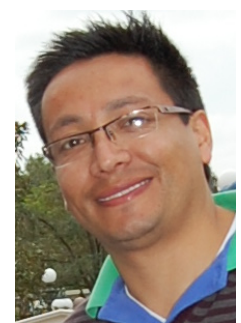

Johann M. Marquez-Barja is a Research Fellow at the CTVR Telecommunications Research Centre at Trinity College Dublin (Ireland). His main research areas of interest are Wireless/Small cells architectures and Passive optical network backhauls. Also, he is also interested on Vehicular Networks, Vertical Handover techniques and Smart cities. He has studied in USA, Bolivia, Cuba and Spain. He holds a Systems Engineering Degree (graduated with honors), a MSc. on Telematics, a MsC on Computer Architecture and a $\mathrm{PhD}$ on Architecture and Technology of Computer Systems issued by the Universitat Politecnica de Valencia (Spain) [graduated with honors cum laude, as well]. On 2004 he received a personal grant from the EU under the Alban Programme to purse the PhD degree. Dr. Marquez-Barja is serving as Editor for two International Journals, as well as being part of several Technical Programme Committees at different worldwide conferences/congresses. At Trinity College Dublin he is also lecturing wireless networks and communications systems.

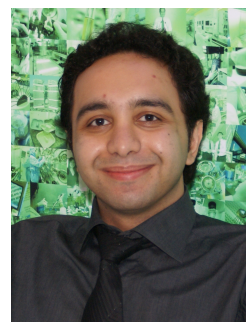

Hamed Ahmadi received his B.Sc. degree in computer engineering from Ferdowsi University of Mashhad, Mashhad, Iran, in 2004, M.Sc. degree in software engineering from National Aerospace University of Kharkiv (KhAI), Kharkiv, Ukraine, in 2008, and $\mathrm{Ph} . \mathrm{D}$. in electrical engineering from National University of Singapore. He was with the R\&D department of Zarrin Afzar electric company, Mashhad, Iran, from 2004 to 2006 . He is currently a research fellow at CTVR, the telecommunications research center, Trinity College Dublin. His research interest includes Machine learning, game theory,Cognitive Radio, dynamic spectrum allocation, radio resource allocation, small-cell networks and selforganizing networks.

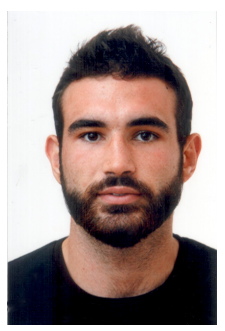

Sergio M. Tornell earned his grade in Telecommunications Engineering by the Universidad Politécnica de Cartagena, Spain in July 2010. In July 2011, after a short experience in the industry, he joined the GRC research group in the Universitat Politècnica de València, Spain. He received his M.Sc. degree in Dec 2011. Currently his is going through a Ph.D in Computer Science. His research interest include Wireless communications, network and mobility modelling, and resource management.

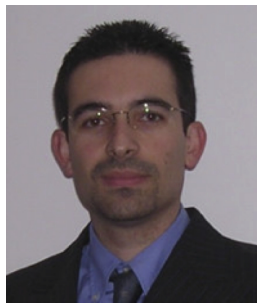

Carlos T. Calafate is an associate professor in the Department of Computer Engineering at the Universitat Politècnica de València (UPV) in Spain. He graduated with honors in Electrical and Computer Engineering at the University of Oporto (Portugal) in 2001. He received his Ph.D. degree in Computer Engineering from the Universitat Politècnica de València in 2006, where he has worked since 2005. He is a member of the Computer Networks research group (GRC). His research interests include mobile and pervasive computing, security and QoS on wireless networks, as well as video coding and streaming. 


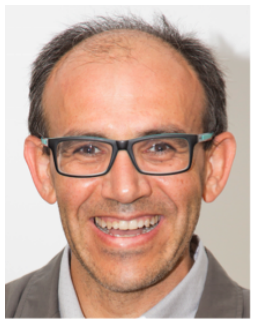

Juan-Carlos Cano is a full professor in the Department of Computer Engineering at the Polytechnic University of Valencia (UPV) in Spain. He earned an MSc and a Ph.D. in Computer Science from the UPV in 1994 and 2002 respectively. From 19951997 he worked as a programming analyst at IBM's manufacturing division in Valencia. His current research interests include Vehicular Networks, Mobile Ad Hoc Networks, and Pervasive Computing.

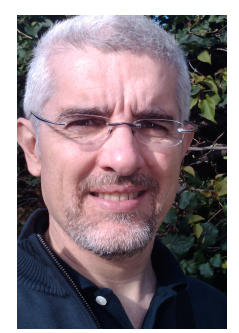

Pietro Manzoni received the MS degree in computer science from the "Università degli Studi" of Milan, Italy, in 1989 , and the $\mathrm{PhD}$ degree in computer science from the "Politecnico di Milano", Italy, in 1995. He is currently a full professor of computer science at the "Universitat Politècnica de València", Spain. His research activity is related to mobile wireless data systems design, modelling, and implementation, particularly oriented to Intelligent Transport Systems. He is a member of the IEEE.

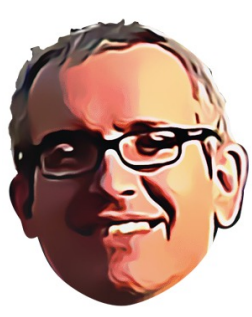

Luiz A. DaSilva is the Professor of Telecommunications at Trinity College Dublin. He is also a Professor in the Bradley Department of Electrical and Computer Engineering at Virginia Tech, USA. His research focuses on distributed and adaptive resource management in wireless networks, and in particular wireless resource sharing, dynamic spectrum access, and the application of game theory to wireless networks. Prof. DaSilva is currently a principal investigator on research projects funded by the National Science Foundation in the United States, the Science Foundation Ireland, and the European Commission under Horizon 2020 and Framework Programme 7. He is a co-principal investigator of CONNECT, the Telecommunications Research Centre in Ireland. 